\title{
interview
}

\section{And then there were two}

\author{
Researchers around the world now have access to a second hard-X-ray free-electron laser facility: the \\ SPring-8 Angstrom Compact Free-Electron Laser in Japan. Nature Photonics spoke to Makina Yabashi and \\ Hitoshi Tanaka from the facility about their achievements and plans for the future.
}

How did SACLA become a reality? In 2001 we wanted to design and build a highperformance, compact X-ray free-electron laser (XFEL) using three key components: a short-period in-vacuum undulator, a high-gradient normal conducting C-band accelerator system and a low-emittance electron injector based on a single-crystal thermionic gun. In 2001-2005 we carried out the basic research and development for these three components (and others), and by 2005 we had already begun constructing a prototype proof-of-principle accelerator. In 2006 we achieved first lasing from this accelerator at ultraviolet wavelengths. Based on this success we obtained funding to construct an XFEL over the next five years from 2006 to 2010, resulting in the SPring-8 Angstrom Compact Free-Electron Laser (SACLA). It took about three months to demonstrate lasing at 1.2 $\AA$. A few days after that we achieved lasing at $1.0 \AA$, and now we have pushed this down to $0.6 \AA$.

Why did you build a new accelerator and develop a new injector design?

The Linac Coherence Light Source (LCLS) at the Stanford Linear Accelerator Center (SLAC) in the USA was a great achievement, and the upcoming European XFEL is another huge project with potentially extremely high performance. However, such large facilities have significant construction and maintenance costs. Another point is that stable and high-performance XFEL operation is a very tricky business. We wanted to avoid issues associated with using existing accelerators or injectors designed for other purposes, such as particle colliders. Our idea was for each component to be designed as part of a practical light source, which would improve the stability of the output beam. This concept, with its reduced costs, should help to proliferate the construction of XFEL facilities around the world.

The gradient of the accelerator at SACLA is very high - around two times that of the LCLS - which allows us to achieve the required beam energy over a shorter distance. In addition, the undulators, which generate light by causing the electrons to wiggle (accelerate), have a very short period. We can achieve shorter lasing wavelengths with

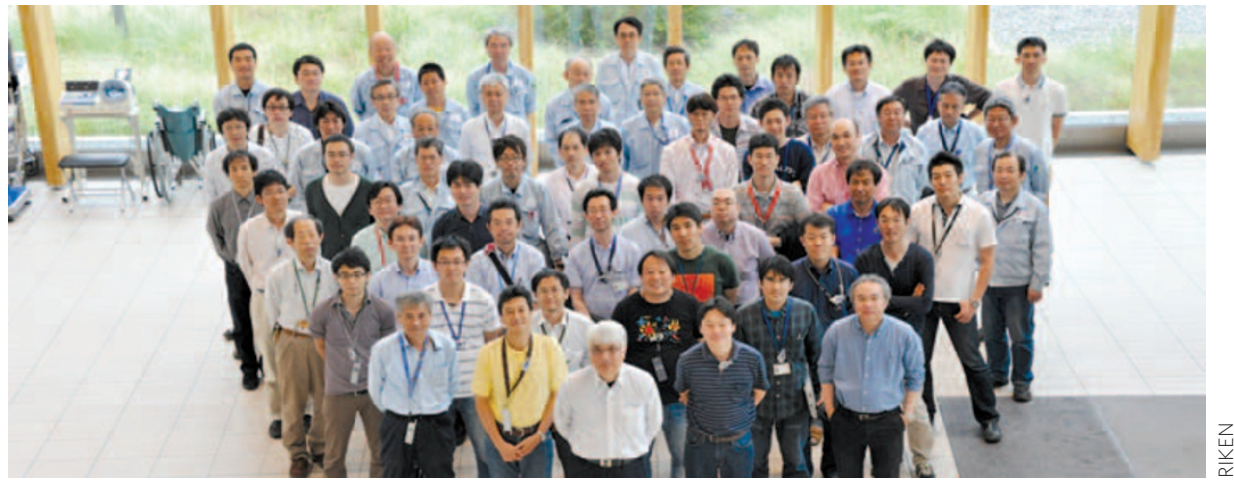

Located $500 \mathrm{~km}$ west of Tokyo, SACLA and its team (pictured) have been providing highly stable hard-X-ray FEL pulses to users from around the world since March 2012.

lower input beam energies than schemes that use longer-period undulators, meaning that the device is smaller for a given emission wavelength. The total length of SACLA is $700 \mathrm{~m}$, which includes a 400-m-long accelerator and $300 \mathrm{~m}$ of undulators and experimental hutches. The facility cost around US $\$ 460$ million to build, which is of the order of the budget for the upgrade project at LCLS-II and much smaller than that of the upcoming European XFEL. Our operating cost is around US\$50 million per year.

Most XFELs launch electrons using a radiofrequency photocathode gun, whereas we decided on a thermionic-emission-based gun. When we were designing the system, the low level of emittance required had not yet been reached, so we developed a new design comprising a single-crystal thermionic gun and a buncher system. The LCLS has actually achieved very low emittance since then; we too could now use a photocathode gun, but this would require more staff to maintain the laser system. We have gone almost a year without any major maintenance on the thermionic gun. However, it should be noted that SACLA requires more radiofrequency sources than the LCLS.

\section{Are you planning to upgrade SACLA?} Scientists around the world currently have access to only two hard-X-ray FEL beamlines: one at the LCLS and another at SACLA. We have the capability to support five XFEL beamlines, and LCLS-II will provide another. Our new beamlines could operate at different wavelengths and have different seeding systems, depending on user requirements and the current state-of-theart technology at the time of upgrade. Our accelerator team has already begun working on the fast path switching of electron pulses $(60 \mathrm{~Hz})$ between the beamlines.

Achieving greater coherence using only a single mode is a primary goal, and for this we may adopt technology similar to that used for self-seeding at the LCLS. This summer we will make space to insert new components for testing seeding schemes by moving the ninth of the 18 undulator segments to the end of the undulator beamline. We are also researching the implemention of highharmonic generation for soft X-rays.

SACLA has a maximum beam energy of about $8.5 \mathrm{GeV}$. Our dark-current removal system at around $3 \mathrm{GeV}$ along the accelerator turned out to be unnecessary, so we could replace this with additional accelerator components to reach a beam energy of $9 \mathrm{GeV}$, which would allow for shorter X-ray emission wavelengths. We are currently limited to wavelengths of $0.6 \AA$, but $0.5 \AA$ or lower may be possible by increasing the electron beam energy.

\section{INTERVIEW BY DAVID PILE}

Makina Yabashi, Hitoshi Tanaka and colleagues have a have a Letter about the successful operation of SACLA and its lasing parameters on page 540 of this issue. 